\title{
Comparison of Two Wake Models for Use in Gradient-based Wind Farm Layout Optimization
}

Jared Thomas

Brigham Young University, jaredthomas68@gmail.com

Eric Tingey

Brigham Young University, ebtingey@gmail.com

Andrew Ning

Brigham Young University, aning@byu.edu

Follow this and additional works at: https://scholarsarchive.byu.edu/facpub

Part of the Mechanical Engineering Commons

\section{Original Publication Citation}

Thomas, J., Tingey, E., and Ning, A., "Comparison of Two Wake Models for Use in Gradient-based Wind Farm Layout Optimization," IEEE Conference on Technologies for Sustainability, Ogden, UT, Jul. 2015. doi:10.1109/SusTech.2015.7314347

\section{BYU ScholarsArchive Citation}

Thomas, Jared; Tingey, Eric; and Ning, Andrew, "Comparison of Two Wake Models for Use in Gradientbased Wind Farm Layout Optimization" (2015). Faculty Publications. 1724.

https://scholarsarchive.byu.edu/facpub/1724 


\title{
Comparison of Two Wake Models for Use in Gradient-based Wind Farm Layout Optimization
}

\author{
Jared Thomas, Eric Tingey, and Andrew Ning \\ Department of Mechanical Engineering \\ Brigham Young Univeristy \\ Provo, Utah, USA \\ Email: jaredthomas@byu.edu
}

\begin{abstract}
Wind farm layout has a significant impact on the productivity of a wind farm. To ensure that the turbines are placed in the most advantageous arrangement, optimization algorithms are often used during the layout design process. Depending on the wake model used for the optimization, optimizing the layout can be time intensive or potentially inaccurate. In this paper we present a comparison of optimization results using two simple wake models, the FLORIS model and the Jensen model. Results highlight some of the key similarities and differences of layout optimization results when different wake models are used.
\end{abstract}

Keywords—wind turbine; wind farm; wake propagation; wake modeling; Jensen; FLORIS; optimization

\section{INTRODUCTION}

To harness large amounts of wind energy in an area, wind turbines are often grouped in wind farms. The layout of the turbines within a wind farm are optimized during the design process to minimize the cost of energy (COE). There are many factors that affect the COE of the wind farm, including landuse, power production, cabling, and noise. For the purposes of this study, only power production was considered in the optimization process.

Wind farms produce power by extracting energy from the wind and converting it to usable power through generators placed in the nacelle of the turbines. As each of the turbines extract energy from the wind they cause a reduction in wind speed and an increase in turbulence in the downwind air. The airflow that is affected by a turbine is called the wake. There is a significant reduction in the power production of turbines that are shadowed by other turbines' wakes. When the power production of turbines within the farm is reduced, the cost of energy is increased. To reduce the effects of shadowing wakes, the turbines must be placed in such a way that the wake effects between turbines is minimized. This leads to the greatest possible power production from the wind farm [1]. Thus, predicting how these wakes propagate, and adjusting the layout accordingly, directly affects the final $\mathrm{COE}$ from the wind farm.

Work on wind farm design optimization has progressed along two basic routes as pointed out by [2]: wake model improvement and numerical solution method development. In order to create better wind farm designs, these tracks need to be combined to improve our understanding of how the results of optimization algorithms differ when different wake models are used. There are two main divisions of optimization methods: gradient-free, and gradient-based. While many wind farm layouts are optimized using gradient-free approaches, gradient-based optimization is preferred due its ability to reliably handle large numbers of design variables while still accurately converging, but it requires gradients of the model being optimized. Both classes of optimization algorithms tend to exploit small differences in the wake models to obtain different solutions that may vary significantly or may not even be optimal (e.g. stopping the optimization at a local minimum or failing to converge). Thus, the suitability of a model for use with optimization, particularly gradient-based optimization, is not necessarily correlated to its accuracy in predicting the power production of a wind farm.

While accurately predicting the total power production of a wind farm is certainly important, the layout of the turbines within the wind farm will ultimately have one of the single greatest impacts on the actual COE of the wind farm [2]. A more accurate model may yield more accurate predictions in terms of power production, but the optimal layout is what will determine the actual COE when a wind farm is built. This means that even if a given wake model does not give accurate predictions of the wind speed in a wake or the power production of a wind turbine, it may still be able to perform a useful layout optimization and determine the best locations for the turbines. Significant savings could be realized in reduced $\mathrm{COE}$ if we can gain a good understanding of how well various wake models function for use in wind farm layout optimization. The final optimized layout determined with a wake model well suited to optimization could then be tested a single time using a high fidelity model to accurately predict what the final power production and COE will be for the wind farm being designed.

Because of the important role that wake models play in wind farm development, there is a concerted effort to define how effective each of the many wake models are so that they may be used with confidence during the wind farm design and analysis process [3]. These efforts include accuracy comparisons of the total power output of a wind farm [3], comparisons for usefulness in control of wind farms [4], and comparisons for wake effect prediction accuracy [5]. However, to our knowledge, a comparison of optimization results using multiple wake models has not yet been performed. 
In this work we present an initial comparison between optimized layouts using two simple models, the FLOw Redirection and Induction in Steady-state (FLORIS) model [6] and the Jensen model [7], [8], demonstrating how the wind farm layout optimization results are affected by using different wake models. This work shows the significant differences in optimized layout that can occur when using models with even small differences. Further research is needed to determine which of the many available wake models performs best with optimization and yields the best layout results.

\section{MODELS}

\section{A. Princess Amalia Wind Farm}

We based our study on the Princess Amalia Wind Farm, located off the coast of the Netherlands. The Princess Amalia Wind Farm consists of 60 turbines placed in parallel rows. The turbines in the farm are Vestas V80 wind turbines with rotor diameters of $80 \mathrm{~m}$. These turbines have a rated power of 2 MW. The original layout can be seen in Fig. 1. Note that this is rotated $90^{\circ}$ from the actual wind farm and then mirrored across the $y$-axis.

For this study, the turbine parameters given in [6] were used instead of those for the actual turbines in the Princess Amalia Wind Farm, which means that we used the NREL 5-MW turbine instead of the actual turbine type used in the Princess Amalia Wind Farm. The change of turbine type was made because of the availability of the necessary turbine parameters for use in the FLORIS model. The rotor diameter of the NREL 5-MW turbines is $126.4 \mathrm{~m}$, which is substantially larger than that of the Vestas V80 turbines in the actual wind farm. Because of this significant difference from reality, our results will not be compared to data from the Princess Amalia Wind Farm.

Wind farm layout optimization requires addressing all wind directions (i.e. using addressing the full wind rose) and using the coordinates of all of the turbines as design variables with multiple constraints. In this work we used wind rose data from the NoordzeeWind meteorological mast that is located near the Princess Amalia Wind Farm. The data was taken before the installation of the OWEZ wind farm, and so the data is undisturbed by that installation. Data was obtained from [9] and is shown in Fig. 2. To simplify the study, and make use of the available tuned parameters for the FLORIS model, only $8 \mathrm{~m} / \mathrm{s}$ wind speeds were addressed. The wind rose data is separated into $5^{\circ}$ segments (resulting in 72 directions), with each direction being weighted according to its frequency for use in the final power calculations. The primary wind direction is from the southwest.

\section{B. The Jensen Model}

The Jensen model is the simpler of the two models used and is only valid in the far wake [7]. There are two versions of the Jensen model presented in [7]. The first uses a single wind speed reduction across the entire wake of a given turbine in the crosswind direction. The second is fit with a cosine function that accounts for the steadily varying conditions across the wake. We used the version fit with the cosine function for

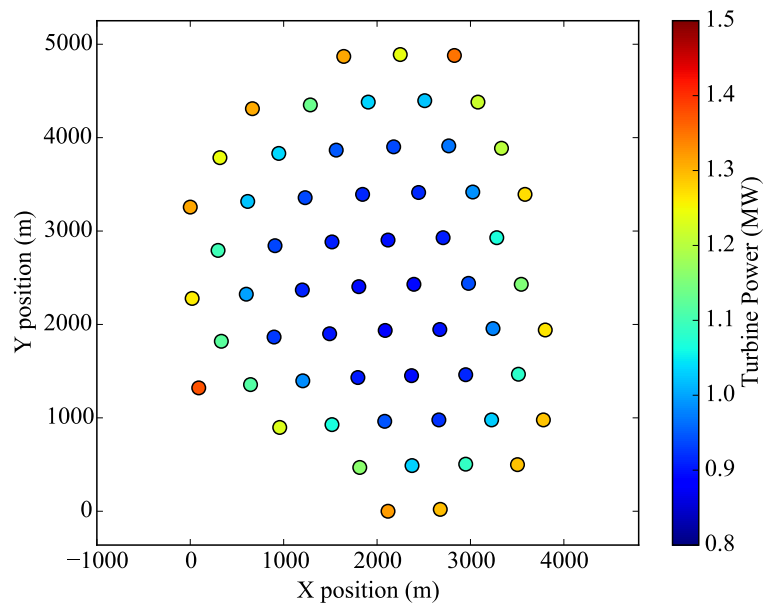

(a) Original locations with power calculations by the Jensen model.

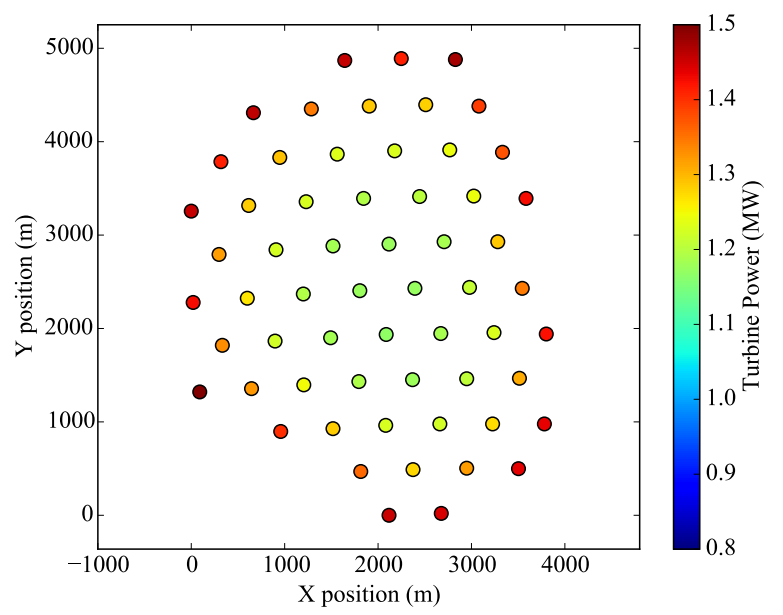

(b) Original locations with power calculations by the FLORIS model.

Fig. 1. Original locations of the turbines in the Princess Amalia Wind Farm. Average power for each turbine is shown as calculated by the FLORIS model and Jensen models respectively. The radii of location markers are not to scale

this study because it provides an approximation of the steadily varying conditions within the wake. Using a model that gives steadily changing wake conditions across the wake is important because the optimization algorithm used relies heavily on gradients as it seeks the best solution. The Jensen model treats a turbine as either completely shadowed by a wake, or not affected at all, but that assumption is softened by the gradual decay of the cosine function mentioned. The power production profile of a turbine at varying positions within a single wake calculated by the Jensen model is shown in Fig. 3. The wakes were combined using the root sum squares method as described in [8].

\section{The FLORIS Model}

The FLORIS model is a derivative of the Jensen model, but is more physically accurate because it accounts for varying conditions in different parts of the wake (known as wake 


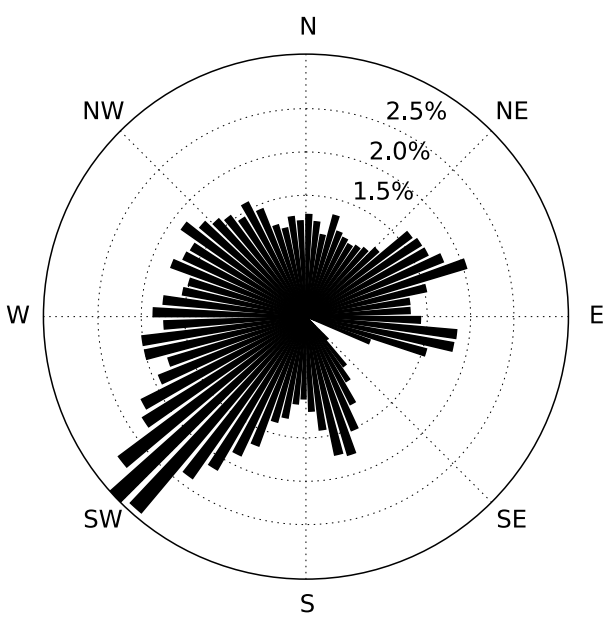

Fig. 2. Windrose from the NoordzeeWind meteorological mast displaying the frequency of wind in each direction [9]. Data shown is only for wind at $8 \mathrm{~m} / \mathrm{s}$ as used in this work.

zones) separately as well as wake offset due to rotor rotation and rotor yaw angle. It also makes simplifying assumptions including an idealized approach to induction across the rotor similar to the Jensen model [6]. The three zones defined by the FLORIS model are the near wake, the far wake, and the mixing zone. Constant wake properties are assumed within each zone at a given downwind position from the wake generating turbine. The varying conditions within the individual zones, perpendicular to the wake center line, are accounted for by using a ratio of the overlap area of the shadowed rotor in each wake zone to the full rotor area. This ratio is then used to calculate the final effective wind speed, which in turn determines the power production of the shadowed turbine and ultimately the COE of the wind farm.

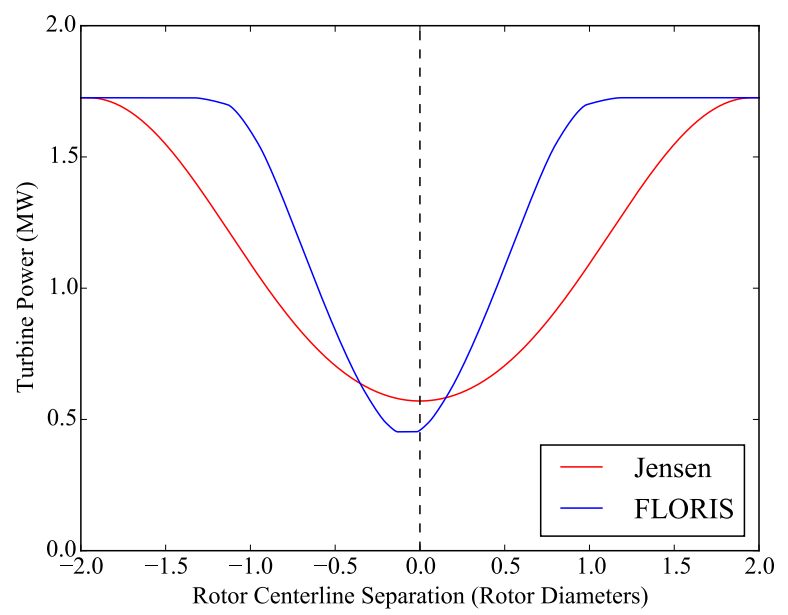

Fig. 3. Power profiles of a turbine shadowed by the wake of single other turbine located 4 rotor diameters upwind using the Jensen and FLORIS models respectively. The dotted line represents the line through the center of the nacelle of the upwind turbine
The FLORIS model combines the various shadowing wakes by using a similar method to the Jensen model. The main difference in the wake combination method is that the overlap ratios are used instead of wind speed deficit.

The power profile of a turbine in the wake of another turbine at various center line separation distances, as calculated by the FLORIS model, can be seen in Fig. 3. The flat spot at the base of the cup, as well as the sharp change in slope towards the lip of the cup are due to the use of the overlap area ratios. When a turbine is completely shadowed by a single wake zone, moving the turbine within that wake zone does not change its power production. When a turbine begins overlapping a new wake zone, the sensitivity of power to the change in relative position also changes. The FLORIS model agrees very well with CFD results as presented in [6].

\section{Comparing the Wake Models}

As shown in Fig. 3, power curves calculated by each of the models of interest are similar, but have some distinct differences. Note that the wake of the FLORIS model is narrower than that of the Jensen model. The wake width affects the optimization process because once a turbine leaves the wake area defined by the model, there is no driving gradient to tell the optimizer to move the turbine any farther from that wake. This is problematic since there may be better locations for the turbine further from that wake. The flat spots present in the FLORIS model create similar difficulties. By contrast, the Jensen model gives a wider and smoother wake definition, and a more gradual return to the non-shadowed state. These elements of the Jensen model are advantageous for use in optimization because they give more positions where the gradients of the model indicate which direction to move each turbine so that a more optimal layout may be found.

Another key difference between the two models, that is not apparent in Fig. 3, is the rate of decay of the wakes. In the FLORIS model, the near wake decays completely, which allows the power of downstream turbines to increase with respect to that recovery. The FLORIS model also accounts for the offset present in the wake due to the rotor rotation and yaw angle with respect to the wind direction. The yaw and decay definitions are key factors for locating turbines within the wind farm (see [10] for yaw optimization results) and are strengths of the FLORIS model.

\section{Optimization Problem DeFinition}

The optimization was performed using SNOPT, a gradientbased sequential quadratic programming optimization algorithm for solving large, nonlinear problems [11]. SNOPT's built in finite difference method was used to obtain gradients. The optimization was defined by (1), where $P(x, y)$ is the overall turbine power output, $\left(x_{i}, y_{i}\right)$ are the $x$ and $y$ locations of each of the turbines in the wind farm reference frame, the separation distance between each pair of turbines $i$ and $j$ is represented by $d_{i, j}$, the rotor diameter of the turbines is denoted by $D_{\text {turbine }}$, and the lower and upper $x$ and $y$ boundaries of the wind farm are denoted by $B_{x, l o w}, B_{x, h i g h}$, $B_{y, l o w}$, and $B_{y, h i g h}$. 


$$
\begin{aligned}
& \underset{x_{i}, y_{i}}{\operatorname{maximize}} P(x, y) \\
& \text { subject to } \quad d_{i, j} \geq 2 D_{\text {turbine }}, \quad i, j=0,1, \ldots, n \\
& B_{x, \text { low }, i} \leq x_{i} \leq B_{x, \text { high }, i}, \quad i=0,1, \ldots, n \\
& B_{y, \text { low }, i} \leq y_{i} \leq B_{y, \text { high }, i}, \quad i=0,1, \ldots, n
\end{aligned}
$$

In this study, boundaries were determined as the smallest rectangle with sides parallel to the $x$ and $y$ axes that would contain the original locations of the turbines in the Princess Amalia Wind Farm.

Separate optimization studies were performed for each model. The power production and location results for each optimization were compared to the baseline as well as power calculations using the other wake model of interest. Power production was compared by percent improvement in reference to the values obtained with the given power calculation model for the original layout. The change in wind farm boundary from the actual wind farm means that the results do no present a fair comparison of optimal layout to original layout. Thus, the comparisons made to the original wind farm are solely for the purpose of comparing optimization results obtained using the individual models.

\section{RESUlts AND Discussion}

We compared the two wake models using the optimized power and locations for each case. This approach provided insight into how the results changed based on the wake model used and highlights some of the key similarities and differences between of the two models investigated.

\section{A. The Jensen Model}

The results obtained using the Jensen model are shown in Fig. 4. Remember that the Jensen model is only valid in the far wake. This means that if turbines are too close in the downwind direction, the results obtained become much less reliable.

Instead of dispersing the turbines evenly throughout the available area, the Jensen model led the optimization to group the turbines into essentially three sets of lines spaced close together, but only rarely activating the turbine separation distance constraint.

The straight lines of turbines are aligned nearly parallel with less frequent wind directions, while being approximately perpendicular to the more frequent wind directions. While this layout is not good for some wind directions, it is very good in the more important directions and thus results in a higher average power production.

\section{B. FLORIS Model}

The layout optimization results obtained using the FLORIS model are shown in Fig. 5. The layout optimized using the FLORIS model is dispersed throughout the domain. However, there are logical patterns in the layout.
First, the turbines are loosely grouped in rows that lie perpendicular to the primary wind direction (from the southwest). Second, the turbines in the southwest corner are spaced further apart than the turbines in the northeast corner to avoid shadowing a large amount of the farm when the wind is from the primary wind direction.

The turbine separation constraint was rarely active during the optimization performed with the FLORIS model, most likely due to the different near wake definition given by the FLORIS model, and its rapid decay in the downwind direction. These characteristics of the FLORIS model force turbines away from close and direct shadowing, but allow shadowing at moderate distances, resulting in a dispersed layout.

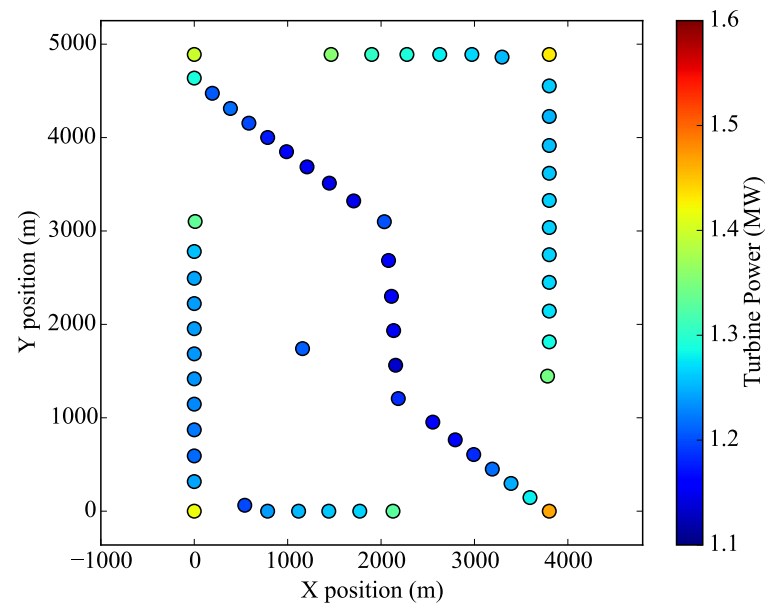

(a) Optimal locations as determined by optimizing with the Jensen model. Power shown was calculated by the Jensen model.

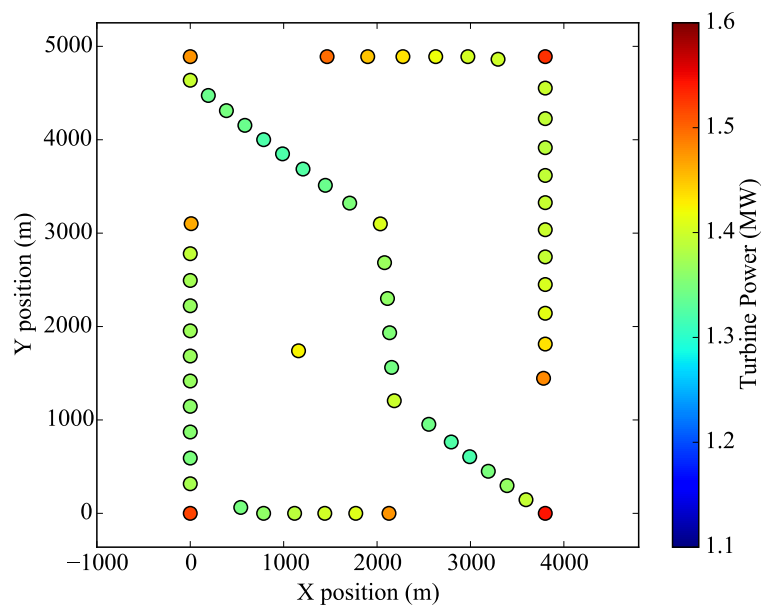

(b) Optimal locations as determined by optimizing with the Jensen model. Power shown was calculated by the FLORIS model.

Fig. 4. Optimized locations for turbines within the Princess Amalia Wind Farm as optimized using the Jensen model. Average power for each turbine is shown as calculated by the FLORIS model and Jensen models respectively. The radii of location markers are not to scale 


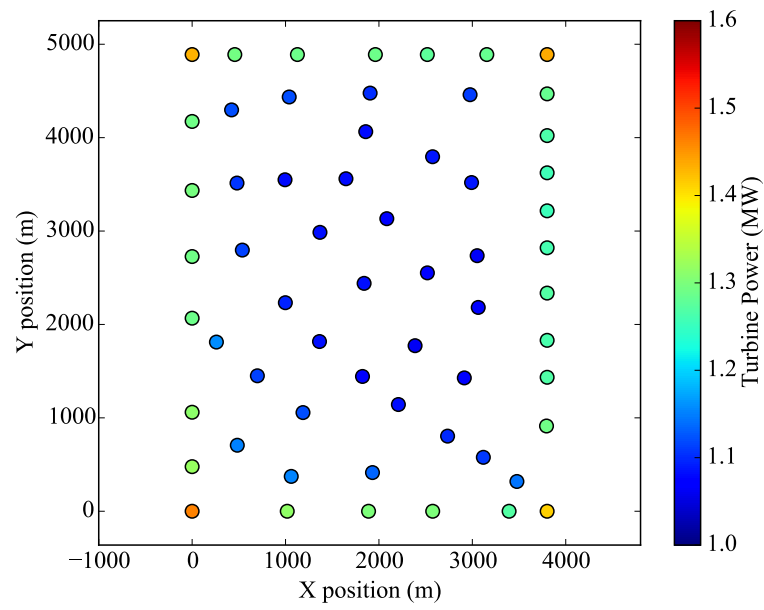

(a) Optimal locations as determined by optimizing with the FLORIS model. Power shown was calculated by the Jensen model.

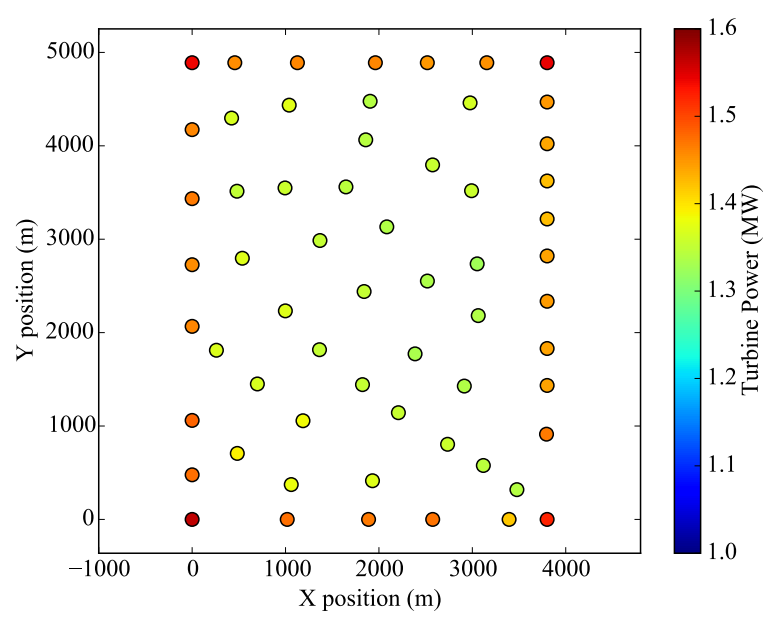

(b) Optimal locations as determined by optimizing with the FLORIS model. Power shown was calculated by the FLORIS model.

Fig. 5. Optimized locations for turbines within the Princess Amalia Wind Farm as optimized using the FLORIS model. Average power for each turbine is shown as calculated by the FLORIS model and Jensen models respectively. The radii of location markers are not to scale

\section{Comparison of Optimization Results}

There are two key similarities between the optimized results. First, the turbine placement along the edges of the wind farm, an intuitive place to put turbines since it increases the overall turbine separation, and thus decreases wake interactions. Second, the development of a line of turbines running from the southeast corner towards a point just below the northwest corner.

The differences between layouts obtained using each of the wake models seems odd at first. However, on closer comparison of the power results, along with an analysis of what is causing the differences, the results begin to make sense. In Table I we see that optimization with each of the models yields good results, spreading the turbines out to utilize
TABLE I. POWER OPTIMIZATION RESUlTS COMPARISON

\begin{tabular}{lccc}
\hline Opt. Model & Evaluation Model & Power Production (MW) & Change (\%) \\
\hline None & Jensen & 63.92 & N/A \\
None & FLORIS & 78.22 & N/A \\
Jensen & Jensen & 77.00 & 20.5 \\
Jensen & FLORIS & 85.46 & 9.3 \\
FLORIS & Jensen & 72.60 & 13.6 \\
FLORIS & FLORIS & 85.36 & 9.1 \\
\hline
\end{tabular}

the available space and increasing the power production of the wind farm from the starting locations, regardless of the power model used to calculate the final power (note that the percent change is in relation to the original layout using the same model for power calculations). The layout optimized with the Jensen model yields higher overall power than the layout optimized with the FLORIS model as calculated by both the FLORIS and Jensen models. While the strong influence of the near wake in the FLORIS model appears to have encouraged a large separation between turbines in all directions, optimizing with the FLORIS model did not find the best solution, as illustrated by the fact the the FLORIS model predicts higher power for the optimal layout obtained using the Jensen model than it does for the optimal layout obtained by the FLORIS model (see Table I).

Another factor keeping the optimization using the FLORIS model from finding a better solution is the narrowness of the FLORIS wake as compared to the Jensen wake as shown in Fig. 3. The narrower FLORIS wake allows turbines to remain in areas of little to no change with small location perturbations due to being outside of nearby wakes (the area outside of a turbine wake is a flat area, meaning that the power gradient in that area is zero). In these cases, the optimizer would consider the location to be optimal and exit the algorithm. A related issue is the use of rotor overlap to the discrete zones (near wake, far wake, and mixing zone) in the FLORIS model. The overlap ratio allows the optimizer to find areas where a turbine is shadowed by one or more wakes, but slight movements yield no improvements due to the turbine rotor area being completely inside a single wake zone. The combination of the two areas of relative flatness in the FLORIS model make it difficult for the optimization algorithm to converge. It is quite possible that if better gradients were obtained for the FLORIS model it would work much better in layout optimization.

\section{CONCLUSION}

To determine which layout truly produces the most power we would need to perform a high-fidelity simulation of both layouts. However, the initial comparison presented here illustrates the differences in optimization layouts resulting from using each of the models. It appears that even though the FLORIS model has a more detailed wake description, the cosine version of the Jensen model is better suited to large-scale gradient-based optimization because of its smooth gradients. Future work will include a comparison of a greater variety of wake models and an analysis of the results using higher fidelity methods to determine which model works most effectively with the optimization algorithm to find the optimal layout. Improving the FLORIS model for use in gradient-based 
optimization will also be considered. The results presented here appear promising because they show that a more detailed model is not inherently better for use in optimizing wind farm layout with gradient-based optimization.

\section{REFERENCES}

[1] J. Dabiri, J. Greer, J. Koseff, P. Mois, and J. Peng, "A new approach to wind energy: opportunities and challenges," in AIP Conference Proceedings, 2015.

[2] J. Herbert-Acero, O. Probst, P. Rethore, G. Larsen, and K. CastilloVillar, "A review of methodological approaches for the design and optimization of wind farms," Energies, vol. 7, no. 11, 2014.

[3] M. Gaumond, P.-E. Réthoré, A. Bechmann, S. Ott, G. C. Larsen, A. Pena Diaz, and K. S. Hansen, "Benchmarking of wind turbine wake models in large offshore windfarms," in Making Torque from the Wind Conference, Oldenburg, Germany, October 2012.

[4] J. Annoni, P. Seiler, K. Johnson, P. Fleming, and P. Gebraad, "Evaluating wake models for wind farm control," in American Control Conference. New York: American Automatic Control Council, June 2014.
[5] S. J. Andersen, J. N. Sørensen, S. Ivanell, and R. F. Mikkelsen, "Comparison of engineering wake models with CFD simulations," Journal of Physics: Conference Series (Online), vol. 524, no. 1, 2014.

[6] P. M. O. Gebraad, F. W. Teeuwisse, J. W. van Wingerden, P. A. Fleming, S. D. Ruben, J. R. Marden, and L. Y. Pao, "Wind plant power optimization through yaw control using a parametric model for wake effects-a CFD simulation study," Wind Energy, 2014.

[7] N. O. Jensen, "A note on wind generator interaction," Ris $\emptyset$ National Laboratory, DK-4000 Roskilde, Denmark, Tech. Rep., November 1983.

[8] I. Katic, J. Højstrup, and N. Jensen, "A simple model for cluster efficiency," in European Wind Energy Association Conference and Exhibition. Rome - Italy: European Wind Energy Association, October 1986.

[9] (2013) NoordzeeWind BV. Data of the NoordzeeWind monitoring and evaluation programme (NSW-MEP). [Online]. Available: http://www.noordzeewind.nl/en/knowledge/reportsdata/

[10] P. Fleming, A. Ning, P. Gebraad, and K. Dykes, "Wind plant system engineering through optimization of layout and yaw control," Wind Energy, 2015.

[11] P. Gill, W. Murray, and M. Saudners, "Snopt: an sqp algorithm for large-scale constrained optimization," SIAM Review, vol. 47, pp. 99$131,2005$. 